\title{
System Identification Methodology Preliminary Research on Maneuvering Motion of a New Type Unmanned Surface Vehicle
}

\author{
Tianyu Ma ${ }^{a}$, Taotao Wang ${ }^{b}$, Jun $\mathrm{Li}^{\mathrm{c}}$ and Songlin Yang ${ }^{\mathrm{d}}$ \\ School of Naval Architecture and Ocean Engineering, Jiangsu University of Science and Technology, \\ Zhenjiang, Jiangsu 212003, China \\ amatianyu1989@126.com, bjust_xztg@163.com, '995536283@qq.com, \\ dysl560516@vip.163.com
}

Keywords: Unmanned surface vehicle (USV), maneuvering motion, turning test, intelligent autonomous system, system identification, VB.NET language.

\begin{abstract}
A new type unmanned surface vehicle (USV) concept is proposed in this paper. Then, the intelligent autonomous vehicle model is designed and fabricated. Self-propelled model test of the new type USV is carried out, which is mainly turning test. According to the turning diameters in various speed and rudder angles under different control voltages and displacements as well as other researchers' experience, the turning motion identification objective function of the new type USV is established. The new type USV maneuvering motion system identification program based on chaotic genetic optimum algorithm is compiled via VB.NET language. The equivalent mathematical model of turning motion is obtained after identified calculating. The reliability of this identification methodology is verified after comparing the identified results with test data, which can provide a kind of technical support in lucubrating the maneuvering motion pattern of the new type USV.
\end{abstract}

\section{Introduction}

With the ship maneuverability criterion issuing by the International Maritime Organization (IMO), there raised a great upsurge in ship maneuverability research around the world. For a USV, a fantastic maneuverability can not only insure to execute tasks more flexible, but also make a contribution to improving its survivability. Considering the International Regulations for Preventing Collisions at Sea (COLREGS), many researchers concentrate on obstacle avoidance and path planning for USV [1-3]. Moreover, a motion planning method of USV under relative coordinates was described [4]. First-order and second-order linear and nonlinear parameters in the high-speed unmanned planning hull's response equations were identified by support vector machines [5]. The maneuverability of a USV under the action of steady wind pressure was simulated [6].

An excellent maneuverability of USV play an important role when swerving, turning around, autonomous obstacle avoidance and entering or leaving a port, which is related to the turning performance significantly. Turning performance of USV is the performance for circular motion by steerage. As typical motion status, stationary turning motion is one of the most vital maneuvering tests. The emphasis of this work is to design and carry out a serious of turning tests, thereafter utilize a system identification methodology based on chaotic genetic optimum algorithm to identify the test data in order to obtain the equivalent mathematical model.

\section{Hull Design and Tests Design}

Hull Design. Nowadays, high performance craft (HPC) made a rapid progress, mainly due to the advantages such as high speed, well seakeeping capability, large capacity, attractive appearance, better economy and security. The regular planning craft is one of the immemorial types among the HPCs. Meanwhile, fabricate and practical quantity of planning craft always rank first among the HPCs in the past few decades. Based on parent ship transformation method, a new type intelligent autonomous USV model is fabricated using the material of glass fiber reinforced plastics. The 
innovative design lie in extending transverse symmetric short wing sheet bodies in the double bilge chine lines from mishap to stern, which is shown in Fig. 1. The purpose of this design mainly has two points: (1) Restrain the lateral splash effectively when high-speed sliding; (2) On the basis of good rapidity, ensure the stabilization and improve navigation stability. Programmable automatic controller (PAC) is chosen to be the core controller. Motor, steering engine, DGPS and three-dimensional electronic compass are installed to be a close loop connection with PAC. The principle parameters of the new type USV model are: Vehicle length $\mathrm{L}=1.5 \mathrm{~m}$; Vehicle breath $\mathrm{B}=0.46 \mathrm{~m}$; Molded depth $\mathrm{D}=0.19 \mathrm{~m}$; Designed draft $\mathrm{T}=0.13 \mathrm{~m}$; Designed displacement $\Delta=50 \mathrm{~kg}$.

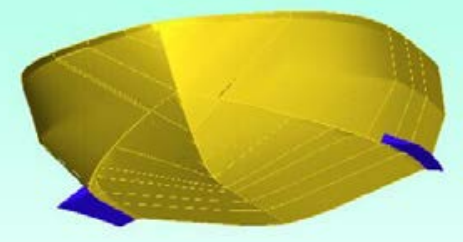

Fig. 1 Effect drawing of the hull with anti-splash sheet bodies

Tests Design. The turning test are carried out in the swimming pool of Jiangsu University of Science and Technology, where the length, width and maximum depth are $50 \mathrm{~m} \times 25 \mathrm{~m} \times 1.9 \mathrm{~m}$. For the convenience of collecting data and ensure the accuracy of the data, turning motion control program of the new type USV is compiled via VB.NET language. The model execute the control program and complete turning motion by the different given rudder angle signal in PAC. The vehicle-mounted DGPS and three-dimensional electronic compass keep a real-time record of test data such as heading angles and speed. In order to study the effect of turning diameters in various speed and rudder angles, multiple composite state turning tests are carried out by adding loads and adjusting motor speed. The rudder angles(left rudder) are $20^{\circ}, 25^{\circ}, 30^{\circ}$ and $35^{\circ}$; The draft are $0.13 \mathrm{~m}, 0.14 \mathrm{~m}$ and $0.145 \mathrm{~m}$; The control voltages are $1.8 \mathrm{~V}, 2.0 \mathrm{~V}$ and $2.2 \mathrm{~V}$.

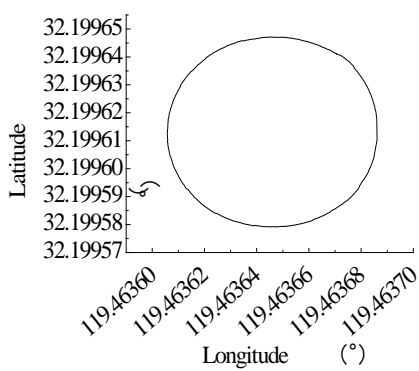

Fig. 2 Trajectory recorded by DGPS

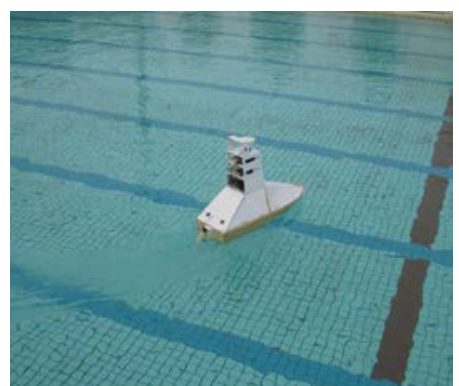

Fig. 3 the turning test

The specific steps for turning test are: (1) Charge the lithium-iron ferrous phosphate storage battery to ensure the power of the model during the test. (2) The turning test control program is debugged on VS2008 platform, set the auto-run after ensuring that the program is correct and storing into PAC. (3) Arrange the DGPS reference station beside the pool, and energize the radio and the receiver. (4) Connect each vehicle-mounted measuring equipment and energizing, start PAC to test operation of the program, and observe positioning state of DGPS. (5) Lunch the model, add loads until the model floating and meet the design draft. (6) Make trial run for 1 2 times, and test the working condition of equipment. (7) Lay a 30m tape on the edge of the pool as the reference mark. (8) After the preparations, turning test starts. Firstly, make the model keep $0^{\circ}$ rudder angle, sail direct for a moment to achieve stable speed. Secondly, give the model different rudder angle signals for turning motion. Speed and real-time position information are recorded by DGPS (Fig. 2).Turning diameters depend on manual measurement. When the model turning stably more than a round to achieve the steady turning state, start to record the turning diameter. When the stem is perpendicular to the pool wall for the first time, record the first numerical value referring to the tape. Similarly, when the stem is perpendicular to the pool wall for the second time, record the second numerical value. Difference of the two numerical value is the turning diameter. (9) After one state test, proceed the next state test after dissipating the surface aftermath (Fig. 3). 
Table 1 the turning test data

\begin{tabular}{|c|c|c|c|c|c|c|c|}
\hline \multirow[t]{3}{*}{ Control Voltage(V) } & \multirow[t]{3}{*}{ Draft(m) } & \multirow[t]{3}{*}{ Displacement(kg) } & \multirow[t]{3}{*}{ Speed(kn) } & \multicolumn{4}{|c|}{ Rudder Angle $\left(^{\circ}\right)$} \\
\hline & & & & 20 & 25 & 30 & 35 \\
\hline & & & & \multicolumn{4}{|c|}{ Turning Diameter(m) } \\
\hline & 0.13 & 50 & 1.06 & 13.05 & 11.15 & 9.4 & 7.85 \\
\hline & 0.14 & 60 & 0.88 & 14.3 & 12.3 & 10.05 & 8.4 \\
\hline \multirow[t]{3}{*}{1.8} & 0.145 & 70 & 0.73 & 12.35 & 11.35 & 9.3 & 7.45 \\
\hline & 0.13 & 50 & 1.21 & 13.75 & 11.25 & 9.55 & 8.15 \\
\hline & 0.14 & 60 & 1.13 & 15.25 & 13.65 & 10.45 & 8.8 \\
\hline \multirow[t]{3}{*}{2.0} & 0.145 & 70 & 1.07 & 14.55 & 11.4 & 9.6 & 8.55 \\
\hline & 0.13 & 50 & 1.4 & 13.35 & 11.1 & 10.1 & 8.4 \\
\hline & 0.14 & 60 & 1.31 & 14.95 & 13.05 & 10.4 & 8.9 \\
\hline 2.2 & 0.145 & 70 & 1.23 & 14.2 & 12.3 & 10.15 & 8.6 \\
\hline
\end{tabular}

\section{Identification Methodology and Analysis}

Identification objective function. How to obtain the equivalent mathematical model of turning motion by using less identification parameters as possible is of great importance, due to the system identification methodology requires a higher precision in measuring the motion parameters. It is not difficult to find in accordance with the test data (Table 1): The turning diameters have no obvious positive correlation or negative correlation with speed, meanwhile the turning diameters have basic negative correlation with rudder angles. Considering the relationship curve of relative turning diameter and Froude number, which refers to a serious of actual ship test and self-propelled model test information [7]. Furthermore, combining the previous study [8], select the form of fitting polynomial to build the identification model with the dimensionless variables:

$$
D_{L}=a_{1} \frac{F r+a_{2} F r^{2}+a_{3} F r^{3}}{\delta^{a_{4}}+a_{5} \delta}
$$

Where, the relative turning diameter $D_{L}=D / L ; D$ is turning diameter; $L$ is the vehicle length; the Froude number $F r=V / \sqrt{g L} ; \delta$ is the rudder angle (the unit is rad); the $a_{1}, a_{2}, a_{3}, a_{4}$ and $a_{5}$ are the identification parameters. Therefore, the identification objective function is:

$$
F=\sqrt{\frac{\sum_{i=1}^{N}\left[D_{L i}-a_{1}\left(F r+a_{2} F r^{2}+a_{3} F r^{3}\right) /\left(\delta^{a_{4}}+a_{5} \delta\right)\right]^{2}}{N}}
$$

Where, $N$ is the number of sets of test data.

Identification process. Preprocess the test data using off-line identification method, then calculate the input and output data. Genetic algorithm (GA) is an adaptive heuristic and global optimization search algorithm, which has some shortcomings such as: local search capabilities and easy to fall into a precocious. Chaos is a general non-linear phenomenon in the nature with randomness, periodicity and regularly, which is intensively sensitive for the initial values. Chaos algorithm (CA) is easy to escape from local minima and happen to make up for the defects of GA. Therefore, use chaotic genetic algorithm (CGA) in the process of identification calculation to obtain the parameters of the model. The new type USV maneuvering motion system identification program based on chaotic genetic optimum algorithm is compiled via VB.NET language, which has the steps as follows:

Set the objective function F. (2) set the range of each identification parameter. (3) Set the relative parameters of CGA. (4) Divide the test data into 3 columns: Fr, $\delta$ and DL and save them into an Excel file, input the file into the program. (5) Click the "Run" button to calculate and observe the coincidence degree in the drawing area on the interface. If the relative error is too big, return to the step (2), adjust the range of each identification parameters and recalculate until the relative error down to quiet low degree.

Identification results and analysis. The range of each identification parameter is determined after repeatedly tentative calculation, which is followed by $a_{1} \in[-20,700], a_{2} \in[-5,5], a_{3} \in[0,5], a_{4}$ 
$\in[-5,5], a_{5} \in[-5,30]$. Set the genetic population size of 100 and the genetic iterations number of 1000 , the crossover probability of 0.8 , the mutation probability of 0.02 , the coding precision of 4 . Set the chaotic iterations number of 100 , chaotic population account for $10 \%$ of the genetic population. It comes to the conclusion that the identification results are comparatively ideal when the selection mode is roulette, the crossover mode is single-point crossover and the coding mode is real coding (Fig 4).

Table 2 Comparison between the system identification results and the test data

\begin{tabular}{|c|c|c|c|c|}
\hline \multicolumn{3}{|c|}{ Input Data } & \multirow{2}{*}{$\begin{array}{c}\text { Output Data } \\
\text { Identification Results DLi }\end{array}$} & \multirow[b]{2}{*}{$\begin{array}{l}\text { Relative } \\
\text { Error }\end{array}$} \\
\hline Froude Number Fr & Rudder Angle $\delta$ & Relative Turning Diameter DL & & \\
\hline 0.1422 & 0.349 & 8.700 & 9.857 & $13.29 \%$ \\
\hline 0.1422 & 0.436 & 7.433 & 7.894 & $6.20 \%$ \\
\hline 0.1422 & 0.524 & 6.267 & 6.584 & $5.06 \%$ \\
\hline 0.1422 & 0.611 & 5.233 & 5.648 & $7.92 \%$ \\
\hline 0.1181 & 0.349 & 9.533 & 9.454 & $0.83 \%$ \\
\hline 0.1181 & 0.436 & 8.200 & 7.571 & $7.67 \%$ \\
\hline 0.1181 & 0.524 & 6.700 & 6.315 & $5.75 \%$ \\
\hline 0.1181 & 0.611 & 5.600 & 5.417 & $3.27 \%$ \\
\hline 0.0979 & 0.349 & 8.233 & 8.747 & $6.23 \%$ \\
\hline 0.0979 & 0.436 & 7.567 & 7.005 & $7.42 \%$ \\
\hline 0.0979 & 0.524 & 6.200 & 5.843 & $5.77 \%$ \\
\hline 0.0979 & 0.611 & 4.967 & 5.012 & $0.91 \%$ \\
\hline 0.1623 & 0.349 & 9.167 & 9.838 & $7.32 \%$ \\
\hline 0.1623 & 0.436 & 7.500 & 7.879 & $5.05 \%$ \\
\hline 0.1623 & 0.524 & 6.367 & 6.571 & $3.21 \%$ \\
\hline 0.1623 & 0.611 & 5.433 & 5.637 & $3.74 \%$ \\
\hline 0.1516 & 0.349 & 10.167 & 9.887 & $2.75 \%$ \\
\hline 0.1516 & 0.436 & 9.100 & 7.918 & $12.99 \%$ \\
\hline 0.1516 & 0.524 & 6.967 & 6.604 & $5.20 \%$ \\
\hline 0.1516 & 0.611 & 5.867 & 5.665 & $3.44 \%$ \\
\hline 0.1436 & 0.349 & 9.700 & 9.865 & $1.70 \%$ \\
\hline 0.1436 & 0.436 & 7.600 & 7.901 & $3.96 \%$ \\
\hline 0.1436 & 0.524 & 6.400 & 6.590 & $2.97 \%$ \\
\hline 0.1436 & 0.611 & 5.7 & 5.653 & $0.83 \%$ \\
\hline 0.1878 & 0.349 & 8.9 & 9.374 & $5.33 \%$ \\
\hline 0.1878 & 0.436 & 7.4 & 7.508 & $1.45 \%$ \\
\hline 0.1878 & 0.524 & 6.733 & 6.262 & $7.00 \%$ \\
\hline 0.1878 & 0.611 & 5.6 & 5.371 & $4.08 \%$ \\
\hline 0.1758 & 0.349 & 9.967 & 9.653 & $3.14 \%$ \\
\hline 0.1758 & 0.436 & 8.7 & 7.731 & $11.14 \%$ \\
\hline 0.1758 & 0.524 & 6.933 & 6.448 & $7.00 \%$ \\
\hline 0.1758 & 0.611 & 5.933 & 5.531 & $6.78 \%$ \\
\hline 0.1650 & 0.349 & 9.467 & 9.812 & $3.64 \%$ \\
\hline 0.1650 & 0.436 & 8.2 & 7.858 & $-4.17 \%$ \\
\hline 0.1650 & 0.524 & 6.767 & 6.554 & $-3.14 \%$ \\
\hline 0.1650 & 0.611 & 5.733 & 5.622 & $-1.94 \%$ \\
\hline
\end{tabular}

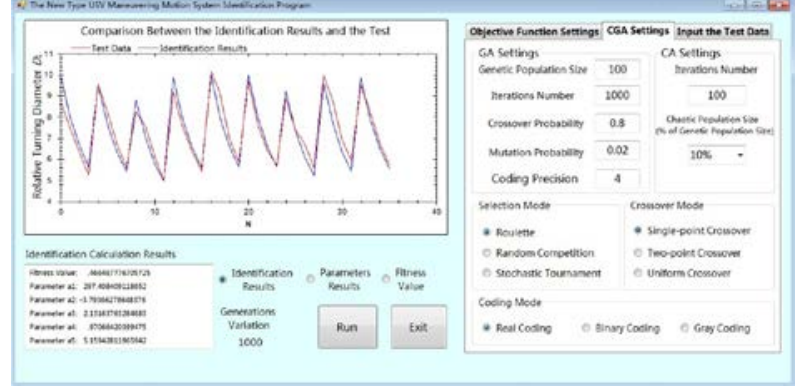

Fig. 4 The program interface of system identification results 
Table 2 shows the system identification output results, in which most of relative error are less than $10 \%$. It is believed that indicating that the system identification program is quiet reliable, then the equivalent mathematical model of turning motion can be obtained:

$$
D_{L}=\frac{297.4084\left(F r-3.7937 F r^{2}+2.1316 F r^{3}\right)}{\delta^{0.9707}+5.1594 \delta}
$$

\section{Summary}

This paper emphasizes the system identification application on the maneuvering motion of a new type USV. The new type USV maneuvering motion system identification program based on chaotic genetic optimum algorithm is compiled via VB.NET language. The equivalent mathematical model of turning motion is obtained after identified calculating. The reliability of this identification methodology is verified after comparing the identified results with test data. In addition, the future work mainly concentrate on how to apply the system identification methodology to forecast the laws in various USV maneuvering tests, including Zigzag test, inertia test, spiral test and inverse spiral test.

\section{Acknowledgments}

This research was financially supported by the National Natural Science of China 51379094, the Graduate Scientific Research and Innovation Project of Jiangsu Province JSXZ14_1092 and the Graduate Innovation Project of Jiangsu University of Science and Technology YCX13S-02 under Grant.

\section{References}

[1] W. Naeem, G.W. Irwin and Aolei Yang, COLREGs-based Collision Avoidance Strategies for Unmanned Surface Vehicles, Mechatronics, vol. 22, p. 669-678, 2012.

[2] S. Campbell, W. Naeem and G.W. Irwin, a Review on Improving the Autonomy of Unmanned Surface Vehicles through Intelligent Collision Avoidance Manoeuvres, Annual Reviews in Control, vol. 36, p. 267-283, 2012.

[3] Hanguen Kim, Donghoon Kim, Jae-Uk Shin, Hyongjin Kim and Hyun Myung, Angular Rate-constrained Path Planning Algorithm for Unmanned Surface Vehicles, Ocean Engineering, vol. 84, p. 37-44, 2014.

[4] Jiayuan Zhuang, Yumin Su, Yulei Liao, and Hanbing Sun, Motion Planning of USV Based on Marine Rules, Procedia Engineering, vol. 15, p. 269-276, 2011.

[5] Gongxing Wu, Study of the Maneuverability and Intelligent Control for Unmanned Surface Vehicle, PhD Dissertation of Harbin Engineering University, 2011(In Chinese).

[6] Weijia Ma, Yongjie Pang, Heng Yang, Chunzheng Ji and Xin Du, Simulation of Maneuverability Performance in the Wind Interference of Unmanned Surface Vehicle, Journal of Wuhan University of Technology (Transportation Science \& Engineering), vol. 36(2), p. 231-234, 238, 2012(In Chinese). [7] Shangyong Fan, Ship Maneuverability, National Defense Industry Press, Beijing, 1988(In Chinese).

[8] Tianyu Ma, Jian Cui and Songlin Yang, Maneuvering and Rolling Coupled Motion Test and Analysis of the Trimaran Model, Ship Science and Technology, vol. 34(11), p. 23-26, 36, 2012(In Chinese). 